•研究报告・

\title{
中国-喜马拉雅三种黄耆属植物与其传粉熊蜂的空 间分布预测
}

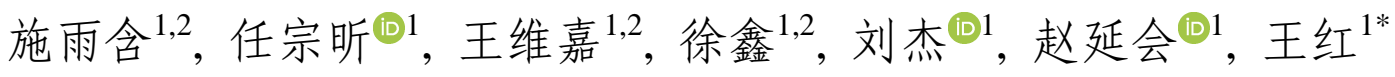

1. 中国科学院昆明植物研究所东亚植物多样性与生物地理学重点实验室, 昆明 650201; 2. 中国科学院大学, 北京 100049

摘要: 依赖于动物传粉获得繁殖成功的植物的分布与其传粉动物的地理分布有着密切联系。预测未来气候变化对植物及其传 粉动物地理分布的影响对生物多样性保护具有重要意义。本文通过对中国-喜马拉雅3种黄耆属(Astragalus)植物, 即弯齿黄者 (A. camptodontus)、黑毛黄耆(A. pullus)和笔直黄耆(A. strictus), 及其传粉熊蜂(Bombus)的野外调查, 以及收集来源于数据库的 黄耆和熊蜂的 543 个物种分布点和 13 个环境因子数据, 结合物种可能出现的完全扩散、不扩散和仅熊蜂扩散 3 种迁移模式, 利 用MaxEnt模型模拟了 3 种黄耆属植物与 2 种传粉熊蜂即橘尾熊蜂(Bombus friseanus)和红束熊蜂(B. rufofasciatus)在历史阶段 (1970-2000年)和2100年两种温室气体浓度情景(ssp245和ssp585)下的适宜分布区变化。结果表明: 3种黄耆属植物均主要依赖 于熊蜂传粉, 黄老与其传粉熊蜂的主要适宜分布区为中国-喜马拉雅地区, 到2100年它们的分布区呈现向西北方向扩张的趋 势, 而在东南部的分布区减少。当模型中考虑与传粉熊蜂的互作后, 3 种黄老属植物的潜在地理分布范围减少了 15.83\%-83.98\%。在温室气体中低浓度情景(ssp245)下，3种黄耆属植物与其传粉熊蜂的空间匹配增加，而在高浓度情景 (ssp585)下弯齿黄耆、黑毛黄耆与橘尾熊蜂的空间匹配降低; 如果物种不扩散或仅熊蜂扩散, 笔直黄耆与红束熊蜂的空间匹配 降低。气候变化和物种的扩散能力可能引起黄耆与其传粉熊蜂出现空间不匹配。同时, 模型预测显示影响黄耆和熊蜂分布的 环境因子不同，但海拔是最主要的环境因子。由于与传粉者的相互作用对许多植物物种的生命周期具有重要意义，因而本研 究可以更好地理解气候变化对植物与其传粉者空间分布的潜在影响，特别是对那些地理范围受限制的植物。

关键词：黄耆属；传粉熊蜂；空间分布；气候变化；最大熵模型；环境因子

施雨含, 任宗昕, 王维嘉, 徐金金, 刘杰, 赵延会, 王红 (2021) 中国-喜马拉雅三种黄耆属植物与其传粉熊蜂的空间分布预测. 生物多样性, 29, 759-769. doi: $10.17520 /$ biods.2020268.

Shi YH, Ren ZX, Wang WJ, Xu X, Liu J, Zhao YH, Wang H (2021) Predicting the spatial distribution of three Astragalus species and their pollinating bumblebees in the Sino-Himalayas. Biodiversity Science, 29, 759-769. doi: 10.17520/biods.2020268.

\section{Predicting the spatial distribution of three Astragalus species and their pollinating bumblebees in the Sino-Himalayas}

\author{
Yuhan Shi ${ }^{1,2}$, Zongxin Ren ${ }^{\left(D_{1}\right.}$, Weijia Wang ${ }^{1,2}$, Xin $\mathrm{Xu}^{1,2}$, Jie Liu ${ }^{\left(\mathbb{D}_{1}\right.}$, Yanhui Zhao ${ }^{\mathbb{D}_{1}}$, Hong Wang ${ }^{1^{*}}$ \\ 1 CAS Key Laboratory for Plant Diversity and Biogeography of East Asia, Kunming Institute of Botany, Chinese Academy of \\ Sciences, Kunming 650201 \\ 2 University of Chinese Academy of Sciences, Beijing 100049
}

\begin{abstract}
Aims: The spatial distribution for plant species that rely on animal pollination for reproduction is influenced by the geographical distribution of their pollinators. Predicting the impact that future climate change will have on the geographical distribution of plants and their pollinators is significantly important for the conservation of biodiversity. Methods: In this study, we conducted a field investigation to map out the distribution for three Astragalus species (A. camptodontus, A. pullus, and A. strictus) and their dominant pollinating bumblebees (Bombus). We collected 543 species distribution points for Astragalus and Bombus as well as 13 environmental factors from online database. Using

收稿日期: 2020-07-03; 接受日期: 2020-08-26

基金项目: 中国科学院先导专项B项目(XDB31000000)、国家自然科学基金-云南省联合重点基金(U1502261)、云南省万人计划云岭学者和青年拔尖人 才项目

* 通讯作者 Author for correspondence. E-mail: wanghong@mail.kib.ac.cn
\end{abstract}


the MaxEnt, we simulated suitable distribution changes for the three Astragalus species and two species of bumblebees (B. friseanus and B. rufofasciatus) under two climate change scenarios for 2100 (ssp245 and ssp585). We also combined with three possible migration situations into the models, i.e. full dispersal, no dispersal and only Bombus dispersal.

Results: We found that three Astragalus species are mainly pollinated by bumblebees and the most suitable distribution for Astragalus and Bombus is the Sino-Himalayas. It is predicted that by 2100, their suitable distribution will expand northwest, while distribution areas in the southeast will decrease. When the plant-pollinator interaction was included in the models, potential range size of the three Astragalus species was reduced by $15.83 \%-83.98 \%$. Under low-emissions scenario (ssp245), the spatial match of three Astragalus species and their pollinating bumblebees is predicted to increase. However, under a high-emissions scenario (ssp585) the spatial match of A. camptodontus, A. pullus and their dominated pollinators $B$. friseanus is predicted to decrease. If species lack full dispersal ability or only Bombus disperse, the spatial match of $A$. strictus and its dominated pollinators $B$. rufofasciatus is predicted to decrease. Climate change and species dispersal ability may cause spatial mismatch between the Astragalus and their pollinating bumblebees. Our simulation shows that the environmental factors affecting the distribution of Astragalus and Bombus are different, but elevation is the most important factor.

Conclusion: Given the importance of pollinators for the life cycle of many plant species, our study could be used to better understand the potential effects of climate change on the spatial distribution of plants and their pollinators, particularly on species that with limited geographical range.

Key words: Astragalus; pollinating bumblebees; spatial distribution; climate change; MaxEnt model; environmental factors

植物-传粉者之间的相互作用是生态系统服务 的重要组成部分(Byers, 2017)。全球气候变化背景 下，一些植物、传粉动物的多样性和种群数量降低, 物种的地理分布也发生改变(Kelly \& Goulden, 2008; Lenoir et al, 2008)。有研究表明, 历史气候变化在空 间上影响了植物与其传粉昆虫的相互作用 (Sosa-Pivatto et al, 2017), 由于植物的迁移能力不如 传粉昆虫, 植物和传粉昆虫之间的迁移速度差异可 能导致它们在地理分布上出现不一致。许多研究采 用物种已知的分布数据和相关环境变量, 通过物种 分布模型(species distribution models, SDMs)预测物 种当前和未来的潜在分布范围(He et al, 2019a, b; Zhang et al, 2019; 张晓玲等, 2019; Li et al, 2020)。 然而物种间的相互作用可能会限制双方的地理分 布(Dufy \& Johnson, 2017; Tsiftsis \& Djordjević, 2020), 尤其是依赖传粉者繁殖的植物, 在评估气候 变化对其分布的影响时应同时考虑传粉者的分布 范围(Gorostiague et al, 2018)。

豆科黄耆属(Astragalus)主要分布于中国-喜马 拉雅、亚洲中部和东北地区。该属植物是牲畜饲料 的重要来源和药用资源, 也为传粉昆虫提供重要的 食源(Clement et al, 2006)。蜂类传粉对黄耆属植物 的繁殖具有重要意义(Green \& Bohart, 1975; Richards, 1987; Tanner et al, 2013), 甚至某些传粉者 也可能是部分黄耆属植物地理分布变迁的限制因
子(Karron, 1987)。熊蜂属(Bombus) 昆虫是北温带生 态系统中最重要的传粉昆虫之一 (Bingham \& Orthner, 1998; Williams et al, 2017)。未来气候变化 可能会影响黄耆属植物与其传粉昆虫的地理分布。 本文以中国-喜马拉雅分布的3种黄耆属植物及其传 粉熊蜂为研究对象, 收集来自于数据库的黄耆属植 物和熊蜂的 543 个物种分布点和 13 个环境因子数据, 结合黄耆和熊蜂可能出现的完全扩散、不扩散和仅 熊蜂扩散 3 种迁移模式, 利用MaxEnt模型预测 3 种 黄耆属植物与 2 种传粉熊蜂在历史阶段和 2100 年两 种温室气体浓度情景(ssp245和ssp585)下的适宜分 布区变化。

\section{1 材料和方法}

\section{1 物种选择}

选择中国西南特有的弯齿黄耆 (Astragalus camptodontus)、黑毛黄耆(A. pullus)和中国-喜马拉 雅分布的笔直黄耆(A. strictus), 及它们的主要传粉 昆虫, 即橘尾熊蜂(Bombus friseanus)和红束熊蜂(B. rufofasciatus)为研究对象(附录1)。基于物种种群的 分布状况, 找出 3 种黄耆属植物与其传粉熊蜂在中 国-喜马拉雅的所有可能适生区域, 选择地理范围 为 $17.9^{\circ}-55.9^{\circ} \mathrm{N}, 60.0^{\circ}-135.3^{\circ} \mathrm{E}$ 的区域(图1)。野外 套袋实验发现3种黄耆没有自交机制，种子生产高 度依赖于传粉昆虫(施雨含等, 未发表数据)。 


\section{2 传粉昆虫调查}

对分布于云南西北部玉龙雪山的弯齿黄耆、黑 毛黄耆各4个居群(3,000-3,300 m), 分布于云南西 北部白马雪山到西藏南部米拉山的 12 个笔直黄耆 居群(3,331-4,760 m)进行野外调查(附录2)。收集样 地植物和昆虫标本, 记录访花数据、传粉昆虫数据 及样地经纬度和海拔信息。对每个样地植物的传粉 昆虫进行调查和记录; 将捕捉到的传粉昆虫放入 $1.5 \mathrm{~mL}$ 离心管中用 $95 \%$ 的酒精浸泡保存。将植物和 昆虫标本带回实验室开展形态鉴定, 对疑难标本利 用DNA 条形码进行辅助鉴定。

\section{3 物种分布数据及处理}

通过在线数据库、相关文献和野外调查数据, 共收集到植物分布数据 1,649 条, 熊蜂分布数据 3,066条。植物分布数据主要有 3 个来源: (1)在线标 本数据平台: 国家标本资源共享平台 (NSII, http://www.nsii.org.cn/2017/home.php)、中国数字植 物标本馆(CVH, http://www.cvh.ac.cn/)、教学标本资 源共享平台(SRSPE, http://mnh.scu.edu.cn/)和全球 生物多样性信息网络(GBIF, https://www.gbif.org/); (2) 中国西南野生种质资源库 (GBOWS, http://www.genobank.org/); (3)野外调查数据(见1.2)。 熊蜂分布数据主要来自全球生物多样性信息网络 (GBIF, https://www.gbif.org/)、野外调查数据(见1.2) 和文献记录(Williams et al, 2009)。

对收集到的数据进行过滤, 去除重复的标本条 目, 以及经纬度和小地点信息都没有的条目。对于 没有经纬度但有详细小地点(记录到乡镇或村)的标 本, 根据记录的小地点和生境、海拔信息, 在
Google Earth上查询对应的经纬度信息。去掉分布信 息与《中国植物志》记载明显不符的条目, 去掉物 种鉴定有误的分布数据。对所有分布数据进行标准 化后按照MaxEnt模型要求进行预处理。为了避免物 种分布数据产生圥余, 用ArcGIS 10.2对物种分布点 做缓冲区分析, 删除5 $\mathrm{km}$ 以内的重复分布点, 得到 最终用于MaxEnt建模的植物分布数据333条, 熊蜂 分布数据210条(图1)。

\section{4 环境变量}

气候数据获取: 从 WorldClim (https://www. worldclim.org/data/cmip6/cmip6climate.html) 下载 Version 2.1版本中代表历史阶段 (Near current: 1970-2000)和代表未来阶段(2100：2081-2100)的分 辨率为 2.5 minutes的 19 个气候变量图层及相同分辨 率的海拔(elevation)数据并提取坡度(slope)和坡向 (aspect) (附录3), 用ArcGIS 10.2根据研究区域进行 掩膜提取, 设置相同的像元大小, 地理坐标统一为 GCS_WGS_1984坐标系。大气环流模型选择国家 (北京)气候中心(The Beijing Climate Center, BCC)的 中等分辨率气候系统模式BCC-CSM2-MR (辛晓歌 等, 2019)。对于2100年的气候图层, 选择两种温室 气体浓度情景: 中低浓度 $\mathrm{CO}_{2}$ 排放情景(ssp245)和 高浓度 $\mathrm{CO}_{2}$ 排放情景(ssp585)。

为了避免在建模过程中气候变量的多重共线 性导致模型过度拟合, 对研究区域19个气候变量图 层进行空间主成分分析, 计算Pearson相关系数(附 录4)。将19个气候因子和物种分布数据导入MaxEnt 3.4.1中运行, 保留模型预测结果中贡献率(percent contribution)大于 $1.0 \%$ 的变量, 当两两相关系数绝

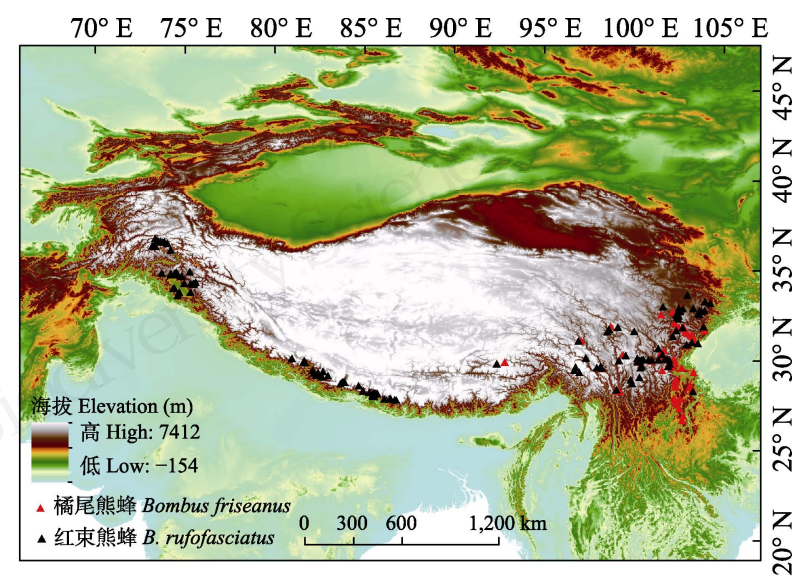

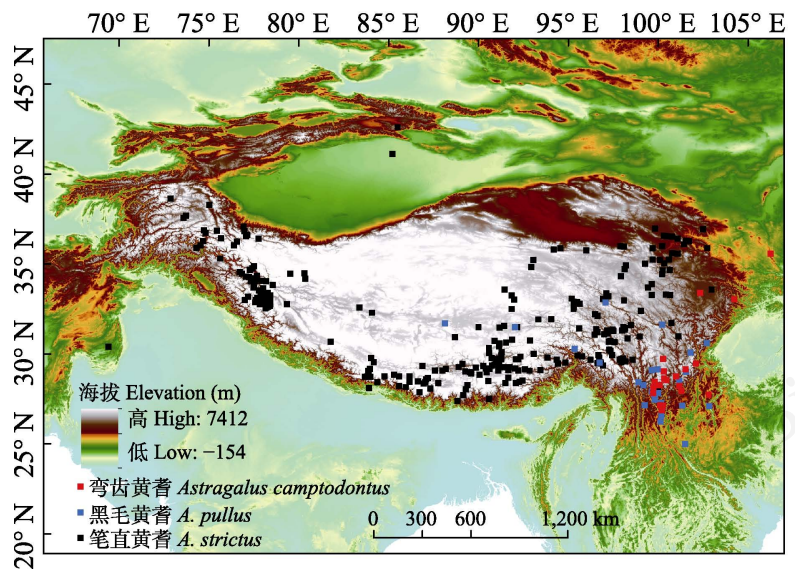

图13种黄耆属植物与其传粉熊蜂的地理分布点

Fig. 1 Occurrence points of three Astragalus species and their pollinating bumblebees 
对值大于 0.8 时, 剔除其中对预测概率贡献较小的 变量(李丽鹤等, 2017), 最终得到用于模型预测的10 个气候因子(附录5): 年平均气温(Bio1)、昼夜温差 月均值(Bio2)、昼夜温差与年温差比值(Bio3)、温度 变化方差(Bio4)、最干季均温(Bio9)、最冷季均温 (Bio11)、年均降水量(Bio12)、最干季降水量(Bio17)、 最热季平均降水量(Bio18)、最冷季平均降水量 (Bio19)和3个地形因子: 海拔、坡向、坡度。

\subsection{MaxEnt参数设置及分析方法}

将物种分布数据和篮选的环境变量导入 MaxEnt 3.4.1软件中运行。设置随机检验百分比 (random test percentage)为25\%, 随机选取 75\%的样 点作为训练集, 用Bootstrap 重复运行 20 次 (和梅香 等, 2018; 史晓昀等, 2019), 其他参数默认设置。使 用ROC (receiver operating characteristic)曲线下的面 积 AUC 值 (area under the receiver operating characteristic curve, AUC)和TSS值(真实技巧统计值, true skill statistic) (Allouche et al, 2006)相结合来评 价模型的优劣。TSS $=$ 灵敏度 $($ sensitivity $)+$ 特异度 (specificity) - 1。灵敏度是实际有分布且被预测为阳 性的概率, 反映模型预测出物种分布的能力; 特异 度是指实际没有该物种分布且被正确预测为阴性 的概率, 反映模型预测该物种没有分布的能力 (王 运生等, 2007)。AUC值和TSS值结合可以更好地评 价模型结果的可信度和准确性, 两者的取值介于-1 到 1 之间。对AUC值而言, $0.60<$ AUC $\leq 0.70$ 表明模 型效果较差; $0.70<\mathrm{AUC} \leq 0.80$ 表明模型效果一般; $0.80<$ AUC $\leq 0.90$ 表明模型效果较好; $0.90<$ AUC $\leq$ 1.00 表明模型效果非常好(Pearce \& Boyce, 2006)。对 TSS值而言, $0.40<\mathrm{TS} \subseteq 0.55$ 表明模型效果较差; $0.55<\mathrm{TSS} \leq 0.70$ 表明模型效果一般; $0.70<\mathrm{TSS} \leq$ 0.85 表明模型效果较好; $0.85<\mathrm{TSS} \leq 1.00$ 表明模型 的模拟效果非常好(Allouche et al, 2006)。

采用刀切法(Jackknife test)和环境因子贡献率 检验各个环境变量对目标物种的重要性, 选择累积 贡献率超过 $90 \%$ 的气候因子为影响物种分布的主导 因子(张晓芹, 2018)。选择MaxEnt输出结果中的10\% 训练存在阈值(10 percentile training presence cloglog threshold值(TH))来定义物种的适生区和非适生区 (Radosavljevic \& Anderson, 2014; Hughes, 2017), 该 阈值使用 $10 \%$ 的物种存在记录的最大值来定义所有 预测最大值较低的区域为物种不存在区域, 而具有
较高值的区域为物种存在区域(Raes et al, 2009)。根 据20次重复运行得到的 cloglog阈值(TH) 和政府间 气候变化专门委员会(IPCC)关于评估物种存在可能 性的划分标准(孙颖等, 2012; 张晓芹, 2018; 张晓芹 等, 2018): $P<0.05$ 为气候不适宜区; $0.05 \leq P<0.33$ 为气候低适宜区; $0.33 \leq P<0.66$ 为气候中适宜区; $P \geq 0.66$ 为气候高适宜区。用ArcGIS 10.2对模型运行 所得的栅格图层重分类, 在20次重复运行的cloglog 阈值(TH)平均值和生境适宜性平均值范围内, 将物 种的潜在分布区分为不适宜区 $(<\mathrm{TH})$ 、适宜区 (TH-0.66) 和高适宜区 $(>0.66) 3$ 类。

用ArcGIS 10.2对历史阶段和未来气候情景下 物种的适宜分布区进行空间变化分析, 确定 3 种黄 耆属植物及其传粉熊蜂分布区中保持不变的区域 (area remaining)、未来扩张的区域 (area to be expended)和未来收缩的区域(area to be lost)。保持下 变的区域指在历史和未来气候情景下物种均有概 率分布的区域; 扩张的区域指在未来气候情景下物 种有概率分布而历史气候条件下无分布的区域; 收 缩的区域指在未来气候情景下物种无分布而在历 史气候情景下有概率分布的区域。

对有互作关系的弯齿黄耆-橘尾熊蜂、黑毛黄 耆-橘尾熊蜂和笔直黄耆-橘尾熊蜂、笔直黄耆-红 束熊蜂的分布区进行叠加分析和面积投影变换, 绘 制每一种黄耆属植物的地理分布范围(Astragalus range, AR)和其传粉熊蜂也存在的区域, 得到它们 的空间匹配范围(spatial match range, SMR)。以SMR 和AR的比值作为衡量黄耆和传粉熊蜂空间匹配的 一个指标, 并考虑未来物种可能出现的 3 种扩散情 况: 物种完全扩散(即物种能够占据模型预测的任 何合适的新栖息地)、物种不扩散(即物种只能占据 原来的栖息地, 不能迁移到新的栖息地)和仅熊蜂 扩散的情况。

\section{结果}

\section{1 黄耆属植物的传粉昆虫组成}

整合2018-2019年野外调查记录的3种黄耆属 植物的传粉昆虫共1,993只(附录1), 包括熊蜂属多 种和中华蜜蜂(Apis cerana), 主要传粉昆虫为熊蜂。 弯齿黄耆的传粉熊蜂共 212 只, 占总数的 $87.24 \%$ 。包 含 9 个形态种, 其中橘尾熊蜂数量最多, 占总数的 $73.66 \%$; 中华蜜蜂共 31 只, 占总数的 $12.76 \%$ 。黑毛 
黄耆的传粉熊蜂共 336 只, 占总数的 $63.52 \%$, 包含 5 个形态种, 其中橘尾熊蜂数量最多, 占总数的 $52.36 \%$; 中华蜜蜂193只, 占总数的 $36.48 \%$ 。笔直 黄 耆的传粉昆虫全为熊蜂, 共1,221只, 包含 19 个形态 种, 其中红束熊蜂数量最多, 占总数的 $34.32 \%$, 其 次为橘尾熊蜂, 占总数的 $22.44 \%$ 。

\section{2 模型评价及环境变量贡献率}

本研究 5 个物种的模型预测结果的AUC平均值 高于 0.95 , TSS平均值高于 0.85 , 表明模型具有较高 的预测能力 (表1)。

对于不同物种, 影响其分布的环境因子不同, 海拔是影响 5 个物种分布最重要的环境因子, 其贡 献率为 $39.20 \%-81.40 \%$ 。除海拔外, 影响弯齿黄耆分 布的主导因子为年均降水量、最冷季均温、昼夜温 差与年温差比值和坡度, 累积贡献率为 $91.60 \%$ 。影 响黑毛黄耆分布的主导因子是最热季平均降水量、 最冷季均温、昼夜温差与年温差比值, 累积贡献率 为 $92.10 \%$ 。影响笔直黄者分布的主导因子是最干季 均温、昼夜温差与年温差比值, 累积贡献率为 $94.70 \%$ 。影响橘尾熊蜂分布的主导因子为最热季平 均降水量、最冷季均温、温度变化方差, 累积贡献 率为 $92.30 \%$ 。影响红束熊蜂分布的主导因子是年均 降水量、最干季均温、最干季降水量, 累积贡献率 为 $90.70 \%$ 。

\section{3 黄耆属植物与其传粉熊蜂的适宜分布区及未} 来气候变化下的分布变化

3 种黄耆属植物与其传粉熊蜂的主要适宜分布 区位于中国-喜马拉雅地区(图2), 弯齿黄耆和黑毛 黄耆的地理分布相似。到2100年, 如果物种完全扩 散, 5 个物种的地理范围都将增加(附录6)。在ssp245 情景下, 3 种黄耆属植物的地理范围增加 14.49\%-63.29\%, 两种熊蜂的地理范围增加 $27.34 \%-53.90 \%$ 。在 ssp585情景下, 3种黄耆属植物 的地理范围增加 $45.53 \%-120.25 \%$, 两种熊蜂的地理
范围增加34.72\%-96.89\%。如果物种不扩散, 它们的 地理范围将减少 $1.50 \%-31.59 \%$ 。

在研究区域内 3 种黄者属植物与其传粉熊蜂的 适宜分布区呈现向西北方向扩张的趋势, 而在东南 部的分布区减少(图2)。在ssp245情景下, 弯齿黄耆、 黑毛黄耆和笔直黄耆将收缩其地理范围的 $16.20 \%$ 、

15.43\%和 $4.15 \%$ 。橘尾熊蜂和红束熊蜂将收缩其地 理范围的 $2.13 \%$ 和 $10.05 \%$ 。在ssp585情景下, 3种黄 耆属植物与其传粉熊蜂地理范围的收缩程度更明 显(附录6)。

\section{4 未来气候变化下黄耆属植物与其传粉熊蜂的 空间匹配范围和空间匹配}

绘制3种黄耆属植物与其传粉熊蜂的空间匹配 范围(SMR), 以及仅有黄耆植物分布的地理范围(图 3)。在物种完全扩散的情况下, 到2100年总的SMR 都增加了(表2)。在ssp245情景下, 3种黄耆属植物与 其传粉熊蜂的 SMR 增加了 $29.70 \%-74.61 \%$, 在 ssp585情景下, SMR增加了 53.50\%-114.70\%。在 ssp245和ssp585情景下, 如果物种不扩散, 到2100 年总的SMR将收缩 $0.99 \%-25.94 \%$ 。如果黄耆不扩散 而熊蜂扩散, 弯齿黄耆与橘尾熊蜂的SMR将减少 $11.53 \%$ 和 $24.56 \%$, 与红束熊蜂的 SMR 将减少 $35.54 \%$ 和 $25.80 \%$ 。

在历史气候情景下 3 种黄耆属植物与其传粉熊 蜂的空间匹配都小于 $80 \%$ (表3)。到2100年, 在 ssp245情景下, 如果物种完全扩散, 它们的空间匹 配将增加 $0.47 \%-23.83 \%$, 在ssp585情景下弯齿黄耆 与橘尾熊蜂的空间匹配将减少 $0.03 \%$, 黑毛黄者与 橘尾熊蜂的空间匹配将减少 $11.79 \%$ 。在ssp245和 ssp585情景下, 如果物种不扩散或仅熊蜂扩散, 笔 直黄者与橘尾熊蜂和红束熊蜂的空间匹配将减少 1.94\%-32.81\%。当考虑传粉熊蜂的地理分布时, 3 种 黄耆属植物的地理分布范围都将减少(图4)。弯齿黄 耆的地理分布将减少 $15.83 \%-23.28 \%$, 黑毛黄耆的

表1 MaxEnt模型结果的受试者工作特征曲线下面积AUC值、真实技巧统计值TSS值和cloglog阈值(TH)

Table1 Area under the receiver operating characteristic curve (AUC), true skill statistic (TSS) and cloglog threshold (TH) of MaxEnt

\begin{tabular}{llll}
\hline 物种 Species & AUC \pm SD & TSS \pm SD & 阈值 Cloglog threshold (TH \pm SD) \\
\hline 弯齿黄耆 Astragalus camptodontus & $0.996 \pm 0.001$ & $0.93 \pm 0.02$ & $0.37 \pm 0.16$ \\
黑毛黄耆 Astragalus pullus & $0.992 \pm 0.001$ & $0.91 \pm 0.02$ & $0.33 \pm 0.11$ \\
笔直黄耆 Astragalus strictus & $0.960 \pm 0.003$ & $0.85 \pm 0.01$ & $0.34 \pm 0.03$ \\
橘尾熊蜂 Bombus friseanus & $0.991 \pm 0.001$ & $0.88 \pm 0.01$ & $0.39 \pm 0.07$ \\
红束熊蜂 Bombus rufofasciatus & $0.985 \pm 0.002$ & $0.87 \pm 0.01$ & $0.32 \pm 0.06$ \\
\hline
\end{tabular}


历史阶段 Near current
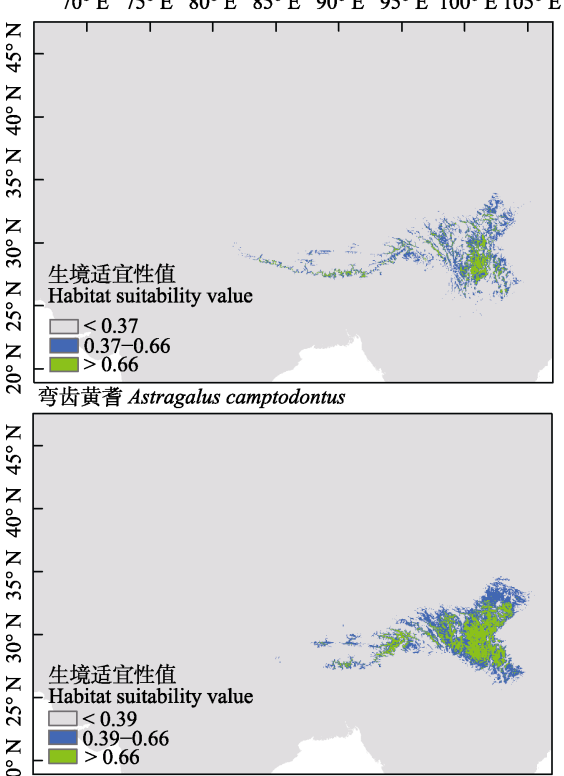

$=0.39-0.66$

黑毛黄硨 A. pullus
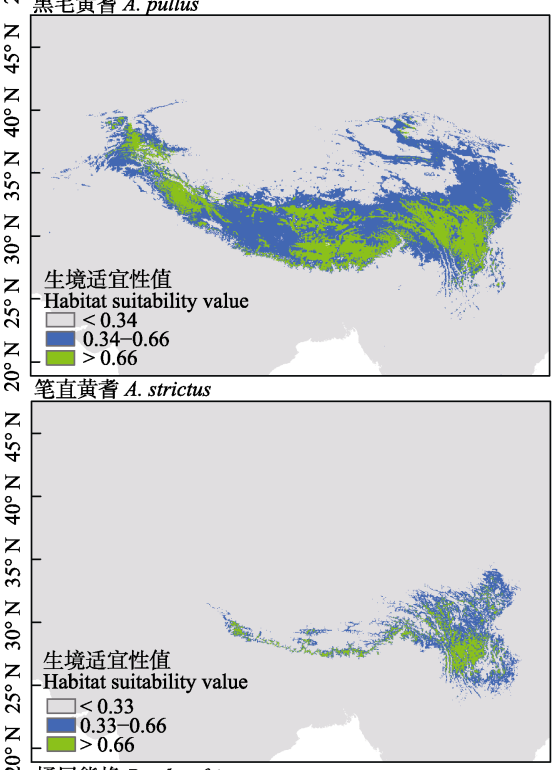

橘尾熊蜂 Bombus friseanus

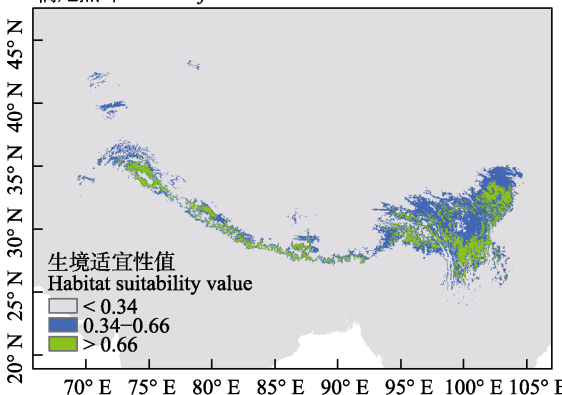

$70^{\circ} \mathrm{E} 75^{\circ} \mathrm{E} 80^{\circ} \mathrm{E}$
红束熊蜂 B. rufofasciatus

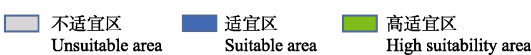

ssp245

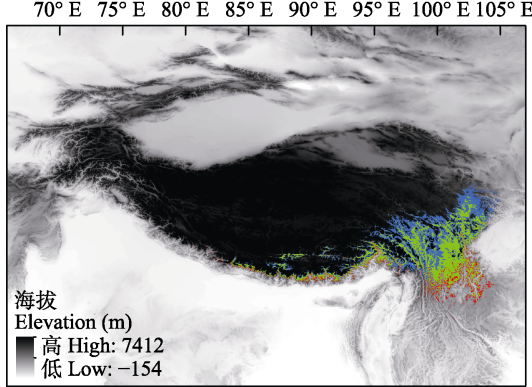

高 High: 7412
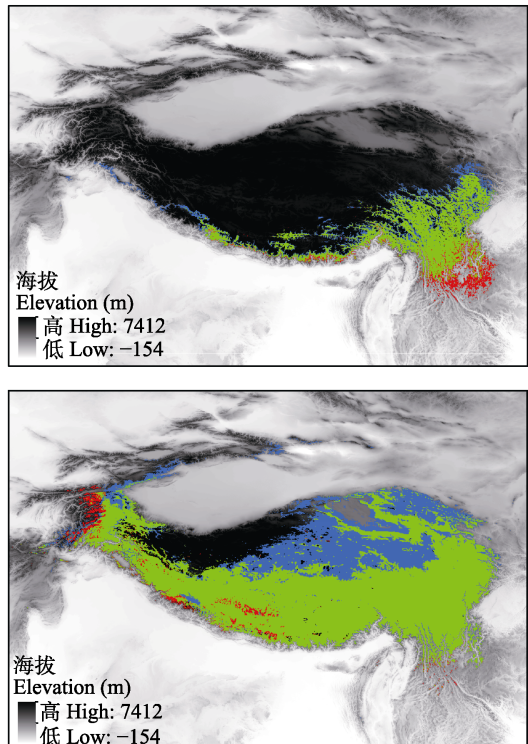

低 Low: -154

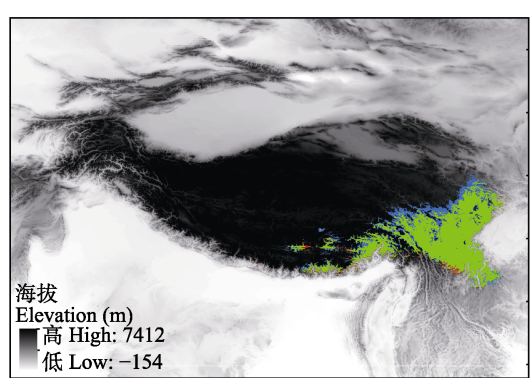

高 High: 7412
低 Low: -154

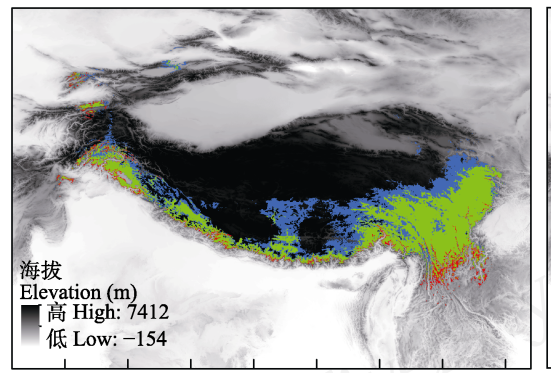

ssp585

$70^{\circ} \mathrm{E} 75^{\circ} \mathrm{E} 80^{\circ} \mathrm{E} 85^{\circ} \mathrm{E} 90^{\circ} \mathrm{E} 95^{\circ} \mathrm{E} 100^{\circ} \mathrm{E} 105^{\circ} \mathrm{E}$
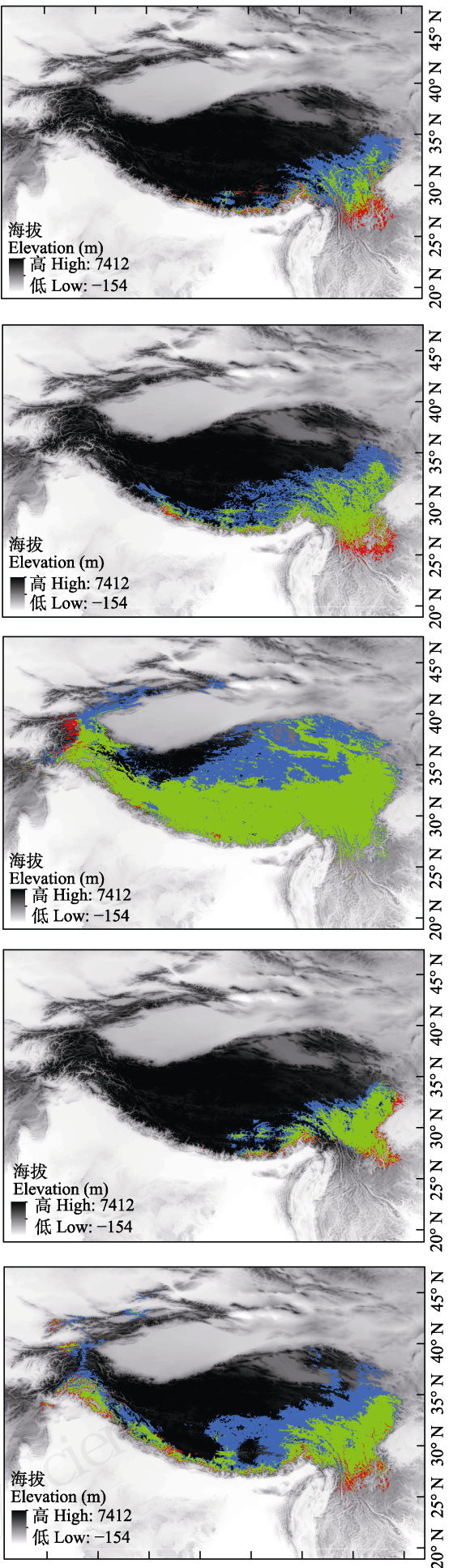

图2 3 种黄茬属植物与其传粉熊蜂在历史阶段(1970-2000年)的适宜分布区和在2100 (2081-2100年)两种情景(ssp245和 ssp585)下的分布变化

Fig. 2 Suitable distribution of three Astragalus species and their pollinating bumblebees at near current (1970-2000) and spatial change at 2100 (2081-2100) two scenarios (ssp245 and ssp585) 

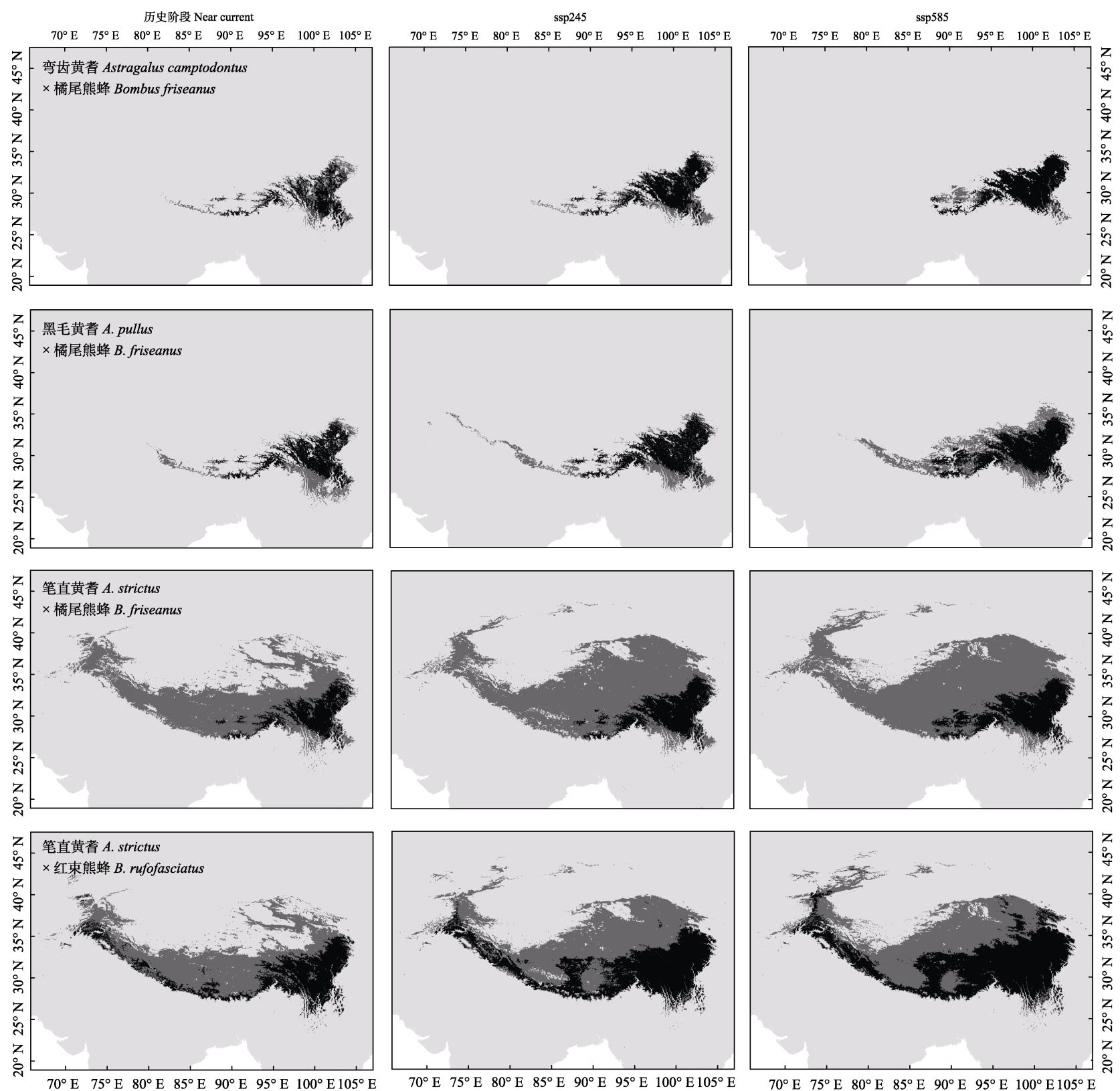

匹配 Match

不匹配 Unmatch

图3 3 种黄老属植物与其传粉熊蜂在历史阶段(1970-2000年)和在2100 (2081-2100年)两种情景(ssp245和ssp585)下的空间匹 配变化。图中黑色部分表示在黄耆分布的区域其传粉者也存在的区域, 灰色部分表示仅黄老分布的区域。

Fig. 3 Change of spatial match of three Astragalus species and their pollinating bumblebees at near current (1970-2000) and at 2100 (2081-2100) two scenarios (ssp245 and ssp585). The black part indicates the area where the pollinator also exists in the plant distributions and the grey part indicates the area where only plants are distributed.

地理分布将减少 $35.14 \%-49.54 \%$ ，笔直黄者的地理 分布将减少 $83.11 \%-83.98 \%$ 。

\section{3 讨论}

植物与传粉者的相互作用关系对维持生态系 统功能完整性具有重要意义, 传粉过程被认为是一
些动植物群体多样化的关键因素(Dodd et al, 1999)。 本研究表明在未来气候变化下3种黄者植物与其传 粉熊蜂地理范围总的趋势是扩大，但其在东南部的 分布区减少。在历史阶段的气候情景下, 3种黄老植 物与其传粉熊蜂在空间上表现出较低的空间匹配 水平 $(<80 \%)$, 在中低浓度 $\mathrm{CO}_{2}$ 排放情景下, 黄者与 
表2 在未来气候变化情景下3种黄耆属植物与其传粉熊蜂的空间匹配范围(SMR)变化百分比。第一列值表示在历史气候情景 下(1970-2000年) 3种黄耆属植物与其传粉熊蜂的SMR。

Table 2 Percentage change in spatially matched range of three Astragalus species and their pollinating bumblebees in the future climate change scenario. First column values indicate the spatially matched range of three Astragalus species and their pollinating bumblebees at near current (1970-2000).

\begin{tabular}{|c|c|c|c|c|c|c|c|}
\hline \multirow{3}{*}{$\begin{array}{l}\text { 物种 } \\
\text { Species (Astragalus } \times \text { pollinators) }\end{array}$} & \multirow{2}{*}{$\begin{array}{c}\text { 空间匹配范围 } \\
\text { SMR } \\
\left(\times 10^{5} \mathrm{~km}^{2}\right)\end{array}$} & \multicolumn{6}{|c|}{ SMR变化百分比 Percentage change in spatially matched range (\%) } \\
\hline & & \multicolumn{2}{|c|}{$\begin{array}{l}\text { 完全扩散 } \\
\text { Full dispersal }\end{array}$} & \multicolumn{2}{|c|}{$\begin{array}{l}\text { 不扩散 } \\
\text { No dispersal }\end{array}$} & \multicolumn{2}{|c|}{$\begin{array}{l}\text { 仅熊蜂扩散 } \\
\text { Only Bombus dispersal }\end{array}$} \\
\hline & $\begin{array}{l}\text { 历史阶段 Near } \\
\text { current }\end{array}$ & ssp245 & ssp585 & ssp245 & ssp585 & ssp245 & ssp585 \\
\hline 弯齿黄耆 × 橘尾熊蜂 & 1.92 & 74.61 & 114.70 & -12.09 & -25.94 & -11.53 & -24.56 \\
\hline $\begin{array}{l}\text { Astragalus camptodontus } \times \text { Bombus friseanus } \\
\text { 黑毛黄耆 } \times \text { 橘尾熊蜂 } \\
\text { Astragalus pullus } \times \text { Bombus friseanus }\end{array}$ & 2.96 & 29.70 & 65.46 & -7.69 & -0.99 & 4.81 & 5.22 \\
\hline $\begin{array}{l}\text { 笔直黄耆 } \times \text { 橘尾熊蜂 } \\
\text { Astragalus strictus } \times \text { Bombus friseanus }\end{array}$ & 3.28 & 32.98 & 53.50 & -1.07 & -3.77 & 225.57 & 294.51 \\
\hline $\begin{array}{l}\text { 笔直黄耆 } \times \text { 红束熊蜂 } \\
\text { Astragalus strictus } \times \text { Bombus rufofasciatus }\end{array}$ & 6.65 & 63.91 & 112.18 & -7.82 & -8.32 & -35.54 & -25.80 \\
\hline
\end{tabular}

表3 在未来气候变化情景下 3 种黄耆属植物与其传粉熊蜂的空间匹配变化百分比。第一列值表示在历史气候情景下 (1970-2000年)与其传粉熊蜂共同分布的黄耆属植物的范围所占的百分比，即空间匹配(\%)。

Table 3 Percentage change in spatial match of three Astragalus species and their pollinating bumblebees in the future climate change scenario. First column values indicate the percentage of the range of each Astragalus species that is shared with its pollinating bumblebees at near current (1970-2000), that is spatial match (\%).

\begin{tabular}{|c|c|c|c|c|c|c|c|}
\hline \multirow{3}{*}{$\begin{array}{l}\text { 物种 } \\
\text { Species (Astragalus × pollinators) }\end{array}$} & \multirow{2}{*}{$\begin{array}{c}\text { 空间匹配 } \\
\text { Spatial match (\%) }\end{array}$} & \multicolumn{6}{|c|}{ 空间匹配变化百分比 Percentage change in spatial match (\%) } \\
\hline & & \multicolumn{2}{|c|}{ 完全扩散 Full dispersal } & \multicolumn{2}{|c|}{ 不扩散 No dispersal } & \multicolumn{2}{|c|}{ 仅熊蜂扩散 Only Bombus dispersal } \\
\hline & $\begin{array}{l}\text { 历史阶段 Near } \\
\text { current }\end{array}$ & ssp245 & ssp585 & ssp245 & ssp585 & ssp245 & ssp585 \\
\hline 弯齿黄耆 × 橘尾熊蜂 & 78.76 & 6.93 & -0.03 & 4.52 & 8.26 & 5.19 & 10.27 \\
\hline Astragalus camptodontus $\times$ Bombus friseanu & & & & & & & \\
\hline $\begin{array}{l}\text { 黑毛黄耆 } \times \text { 橘尾熊蜂 } \\
\text { Astragalus pullus } \times \text { Bombus friseanus }\end{array}$ & 57.22 & 13.28 & -11.79 & 8.44 & 6.03 & 23.12 & 23.82 \\
\hline $\begin{array}{l}\text { 笔直黄耆 } \times \text { 橘尾熊蜂 } \\
\text { Astragalus strictus } \times \text { Bombus friseanus }\end{array}$ & 16.03 & 0.47 & 0.55 & 3.13 & -1.94 & 239.36 & 301.99 \\
\hline $\begin{array}{l}\text { 笔直黄耆 } \times \text { 红束熊蜂 } \\
\text { Astragalus strictus } \times \text { Bombus rufofasciatus }\end{array}$ & 32.51 & 23.83 & 45.80 & -7.82 & -30.74 & -32.81 & -24.39 \\
\hline
\end{tabular}
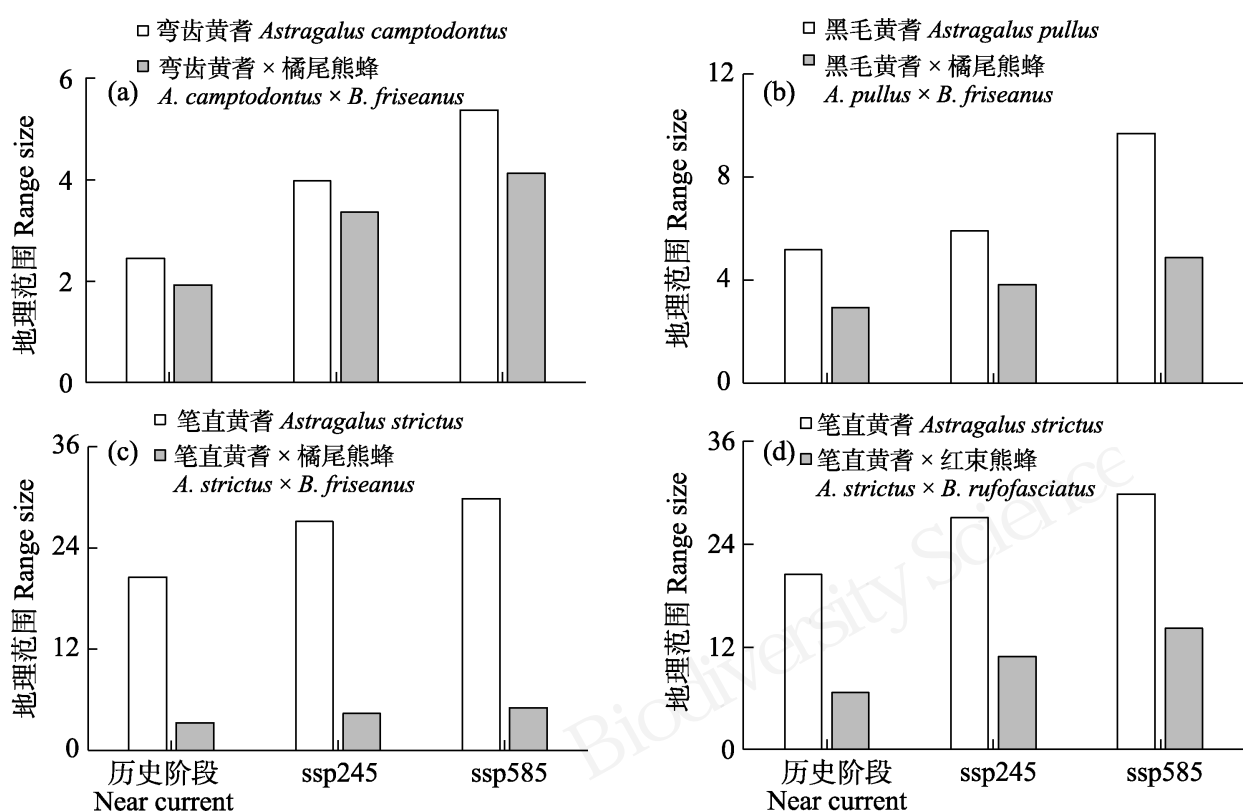

图4 3种黄耆属植物在历史阶段(1970-2000年)和在2100 (2081-2100年)两种情景(ssp245和ssp585)下的地理范围及考虑传粉 熊蜂时的地理范围 $\left(\times 10^{5} \mathbf{k m}^{2}\right)$ 。

Fig. 4 Range size $\left(\times 10^{5} \mathrm{~km}^{2}\right)$ of three Astragalus species and considering the geographical distribution of pollinating bumblebees at near current (1970-2000) and at 2100 (2081-2100) two scenarios (ssp245 and ssp585). 
熊蜂的空间匹配范围和空间匹配度(完全扩散)均增 加, 表明在这种气候变化情景下黄耆与其传粉熊蜂 的空间匹配没有受到影响, 但在高浓度 $\mathrm{CO}_{2}$ 排放情 景下, 有 2 种黄耆植物与其传粉熊蜂的空间匹配降 低, 这表明高强度的气候变化会引起黄耆与其传粉 熊蜂出现空间不匹配。在缺乏传粉者的分布范围, 两者之间原有的互作关系可能会被打破, 当模型中 考虑与传粉熊蜂的互作后, 黄耆的地理分布受到了 其传粉熊蜂的限制。虽然传粉者的专一性和有效性 在不同的区域内是可变的, 一些特化的传粉者消失 后, 一些泛化的传粉者能够代替其传粉, 但植物的 繁殖仍会受到原有传粉者消失的影响(Burkle \& Alarcón, 2011)。

值得关注的是, 气候变化可能破坏物种营养级 上的相互作用, 因为物种之间并非以类似的方式对 这种变化作出反应。从海拔梯度上, 温度变化引起 的物种分布的空间变化更容易观测到。已有研究表 明, 温度升高导致低海拔地区的物种向高海拔地区 迁移的现象较为普遍(Johnson et al, 2010)。我们的研 究表明, 海拔是影响3种黄耆属植物与其传粉熊蜂 地理分布的主要环境因子, 此外, 降水量和极端低 温也在一定程度上影响两者的地理分布。在气候条 件发生变化的情况下, 物种可能适应新的环境(例 如, 通过短期的自然选择)或迁移到新的环境中, 如 果物种无法适应环境变化可能会导致局部灭绝 (Parmesan, 2006)。如果黄耆属植物与其传粉熊蜂不 扩散, 物种只维持它们目前所占据的地理范围的一 部分, 则其地理范围和与其传粉熊蜂的空间匹配范 围将受到限制。如果考虑黄耆不扩散而仅熊蜂扩散 的情况, 笔直黄耆与其传粉昆虫的空间匹配水平降 低。若物种迁移能力弱或由于地理障碍等因素, 物 种间的相互作用受到影响, 可能会增加物种灭绝的 风险。特别是在高浓度 $\mathrm{CO}_{2}$ 排放情景下, 物种受到 的负面影响更强。综上所述, 气候变化可能引起黄 耆植物与其传粉熊蜂出现空间不匹配, 如果物种迁 移到新的分布区, 能否建立新的传粉关系尚不清 楚。因此, 需要进一步加强多因素综合作用对植物传粉昆虫互作关系的影响研究。

致谢: 感谢中国科学院昆明植物研究所丽江森林生 态系统定位研究站提供大力支持; 感谢Paul H.
Williams教授在熊蜂鉴定方面给予的帮助和Peter Bernhardt教授及Judith Trunschke博士对本文提出的 宝贵意见。

\section{ORCID}

任宗昕 (D) https://orcid.org/0000-0001-7265-065X 刘杰 (D) https://orcid.org/0000-0003-4356-1943

赵延会 (D) https://orcid.org/0000-0002-7569-4591

\section{参考文献}

Allouche O, Tsoar A, Kadmon R (2006) Assessing the accuracy of species distribution models: Prevalence, Kappa and the true skill statistic (TSS). Journal of Applied Ecology, 43, 1223-1232.

Bingham RA, Orthner AR (1998) Efficient pollination of alpine plants. Nature, 391, 238-239.

Burkle LA, Alarcón R (2011) The future of plant-pollinator diversity: Understanding interaction networks across time, space, and global change. American Journal of Botany, 98, 528-538.

Byers DL (2017) Studying plant-pollinator interactions in a changing climate: A review of approaches. Applications in Plant Sciences, 5, 1700012.

Clement SL, Griswold TL, Rust RW, Hellier BC, Stout DM (2006) Bee associates of flowering Astragalus and Onobrychis genebank accessions at a Snake River site in eastern Washington. Journal of the Kansas Entomological Society, 79, 254-260.

Dodd ME, Silvertown J, Chase MW (1999) Phylogenetic analysis of trait evolution and species diversity variation among angiosperm families. Evolution, 53, 732-744.

Duffy KJ, Johnson SD (2017) Specialized mutualisms may constrain the geographical distribution of flowering plants. Proceedings of the Royal Society B: Biological Sciences, 284, 20171841.

Gorostiague P, Sajama J, Ortega-Baes P (2018) Will climate change cause spatial mismatch between plants and their pollinators? A test using Andean cactus species. Biological Conservation, 226, 247-255.

Green TW, Bohart GE (1975) The pollination ecology of Astragalus cibarius and Astragalus utahensis (Leguminosae). American Journal of Botany, 62, 379-386.

He MX, Chen LX, Luo G, Gu XD, Wang G, Ran JH (2018) Suitable habitat prediction and overlap analysis of two sympatric species, giant panda (Ailuropoda melanoleuca) and Asiatic black bear (Ursus thibetanus) in Liangshan Mountains. Biodiversity Science, 26, 1180-1189. (in Chinese with English abstract) [和梅香, 陈霍心, 罗概, 古 晓东, 王戈, 苒江洪 (2018) 凉山山系大熊猫和黑熊适宜 生境预测及重叠分析. 生物多样性, 26, 1180-1189.]

He X, Burgess KS, Gao LM, Li DZ (2019a) Distributional 
responses to climate change for alpine species of Cyananthus and Primula endemic to the Himalaya-Hengduan Mountains. Plant Diversity, 41, 26-32.

He X, Burgess KS, Yang XF, Ahrends A, Gao LM, Li DZ (2019b) Upward elevation and northwest range shifts for alpine Meconopsis species in the Himalaya-Hengduan Mountains region. Ecology and Evolution, 9, 4055-4064.

Hughes AC (2017) Mapping priorities for conservation in Southeast Asia. Biological Conservation, 209, 395-405.

Johnson DM, Büntgen U, Frank DC, Kausrud K, Haynes KJ, Liebhold AM, Esper J, Stenseth NC (2010) Climatic warming disrupts recurrent alpine insect outbreaks. Proceedings of the National Academy of Sciences, USA, 107, 20576-20581.

Karron JD (1987) The pollination ecology of co-occuring geographically restricted and widespread species of Astragalus (Fabaceae). Biological Conservation, 39, 179-193.

Kelly AE, Goulden ML (2008) Rapid shifts in plant distribution with recent climate change. Proceedings of the National Academy of Sciences, USA, 105, 11823-11826.

Lenoir J, Gégout JC, Marquet PA, de Ruffray P, Brisse H (2008) A significant upward shift in plant species optimum elevation during the 20th century. Science, 320, 1768-1771.

Li LH, Liu HY, Lin ZS, Jia JH, Liu X (2017) Identifying priority areas for monitoring the invasion of Solidago canadensis based on MaxEnt and Zonation. Acta Ecologica Sinica, 37, 3124-3132. (in Chinese with English abstract) [李丽鹤, 刘会玉, 林振山, 贾俊鹤, 刘翔 (2017) 基于 MaxEnt和Zonation的加拿大一枝黄花入侵重点监控区确 定. 生态学报, 37, 3124-3132.]

Li YC, Li MY, Li C, Liu ZZ (2020) Optimized MaxEnt model predictions of climate change impacts on the suitable distribution of Cunninghamia lanceolata in China. Forests, 11, 302.

Parmesan C (2006) Ecological and evolutionary responses to recent climate change. Annual Review of Ecology, Evolution, and Systematics, 37, 637-669.

Pearce JL, Boyce MS (2006) Modelling distribution and abundance with presence-only data. Journal of Applied Ecology, 43, 405-412.

Radosavljevic A, Anderson RP (2014) Making better MaxEnt models of species distributions: Complexity, ovefitting and evaluation. Journal of Biogeography, 41, 629-643.

Raes N, Roos MC, Slik JWF, van Loon EE, ter Steege H (2009) Botanical richness and endemicity patterns of Borneo derived from species distribution models. Ecography, 32, 180-192.

Richards KW (1987) Diversity, density, efficiency, and effectiveness of pollinators of Cicer milkvetch, Astragalus cicer L. Canadian Journal of Zoology, 65, 2168-2176.

Shi XY, Shi XG, Hu Q, Guan TP, Fu Q, Zhang J, Yao M, Li S (2019) Evaluating the potential habitat overlap and predation risk between snow leopards and free-range yaks in the Qionglai Mountains, Sichuan. Biodiversity Science, 27, 951-959. (in Chinese with English abstract) [史晓昀, 施小 刚, 胡强, 官天培, 付强, 张剑, 姚蒙, 李晟 (2019) 四川 邛崃山脉雪豹与散放牦牛潜在分布重叠与捕食风险评估. 生物多样性, 27, 951-959.]

Sosa-Pivatto M, Cosacov A, Baranzelli MC, Iglesias MR, Espíndola A, Sérsic AN (2017) Do 120,000 years of plant-pollinator interactions predict floral phenotype divergence in Calceolaria polyrhiza? A reconstruction using species distribution models. Arthropod-Plant Interactions, 11, 351-361.

Sun Y, Qin DH, Liu HB (2012) Introduction to treatment of uncertainties for IPCC Fifth Assessment Report. Climate Change Research, 8, 150-153. (in Chinese) [孙颖, 秦大河, 刘洪滨 (2012) IPCC第五次评估报告不确定性处理方法 的介绍. 气候变化研究进展, 8, 150-153.]

Tanner DA, Clark C, Pitts JP (2013) Pollination biology of Astragalus phoenix (Fabaceae) with notes on the natural history of its pollinator, Anthophora porterae (Hymenoptera: Apidae). Western North American Naturalist, 73, 373-381.

Tsiftsis S, Djordjević V (2020) Modelling sexually deceptive orchid species distributions under future climates: The importance of plant-pollinator interactions. Scientific Reports, 10, 10623.

Wang YS, Xie BY, Wan FH, Xiao QM, Dai LY (2007) Application of ROC curve analysis in evaluating the performance of alien species' potential distribution models. Biodiversity Science, 15, 365-372. (in Chinese with English abstract) [王运生, 谢丙炎, 万方浩, 肖启明, 戴良英 (2007) ROC曲线分析在评价入侵物种分布模型中的应用. 生物多样性, 15, 365-372.]

Williams PH, Huang JX, An JD (2017) Bear wasps of the Middle Kingdom: A decade of discovering China's bumblebees. Antenna, 41, 21-24.

Williams PH, Tang Y, Yao J, Cameron S (2009) The bumblebees of Sichuan (Hymenoptera: Apidae, Bombini). Systematics and Biodiversity, 7, 101-189.

Xin XG, Wu TW, Zhang J, Zhang F, Li WP, Zhang YW, Lu YX, Fang YJ, Jie WH, Zhang L, Dong M, Shi XL, Li JL, Chu M, Liu QX, Yan JH (2019) Introduction of BCC models and its participation in CMIP6. Climate Change Research, 15, 533-539. (in Chinese with English abstract) [辛晓歌, 吴统文, 张洁, 张芳, 李伟平, 张艳武, 路屹雄, 房永杰, 颉卫华, 张莉, 董敏, 史学丽, 李江龙, 储敏, 刘 茜霞, 颜京辉 (2019) BCC模式及其开展的CMIP6试验介 绍. 气候变化研究进展, 15, 533-539.]

Zhang L, Jing ZN, Li ZY, Liu Y, Fang SZ (2019) Predictive modeling of suitable habitats for Cinnamomum camphora (L.) Presl using MaxEnt model under climate change in China. International Journal of Environmental Research and Public Health, 16, 3185. 
Zhang XL, Li YC, Wang YY, Cai HY, Zeng H, Wang ZH (2019) Influence of future climate change in suitable habitats of tea in different countries. Biodiversity Science, 27, 595-606. (in Chinese with English abstract) [张晓玲, 李亦超, 王芸芸, 蔡宏宇, 曾辉, 王志恒 (2019) 未来气 候变化对不同国家茶适宜分布区的影响. 生物多样性, 27, 595-606.]

Zhang XQ (2018) Geographical Distribution and Climatic Suitability of Typical Eco-economical Tree Species in the Dryland of Northwest China. PhD dissertation, Research Center for Eco-Environments and Soils and Water Conservation, Chinese Academy of Sciences \& Ministry of
Education, Xi'an. (in Chinese with English abstract) [张晓 芹 (2018) 西北旱区典型生态经济树种地理分布与气候 适宜性研究. 博士学位论文, 中国科学院-教育部水土保 持与生态环境研究中心, 西安.]

Zhang XQ, Li GQ, Du S (2018) Predicting the influence of future climate change on the suitable distribution areas of Elaeagnus angustifolia. Chinese Journal of Applied Ecology, 29, 3213-3220. (in Chinese with English abstract) [张晓芹, 李国庆, 杜盛 (2018) 未来气候变化对沙菄适 宜分布区的影响预测. 应用生态学报, 29, 3213-3220.]

(责任编委: 张大勇 责任编辑: 问文杰)

\section{附录 Supplementary Material}

\section{附录13种黄耆属植物传粉昆虫的种类及数量}

Appendix 1 Species and number of pollinators of three Astragalus species https://www.biodiversity-science.net/fileup/PDF/2020268-1.pdf

\section{附录2 高山草甸样地信息}

Appendix 2 Information of alpine meadow plots https://www.biodiversity-science.net/fileup/PDF/2020268-2.pdf

\section{附录3 WorldClim的环境变量}

Appendix 3 Environment variable from WorldClim https://www.biodiversity-science.net/fileup/PDF/2020268-3.pdf

\section{附录4 19个气候变量的相关性矩阵}

Appendix 4 Correlation matrix of 19 climate variables https://www.biodiversity-science.net/fileup/PDF/2020268-4.pdf

\section{附录5 用于MaxEnt模型的相关气候变量}

Appendix 5 Climate variables for MaxEnt model https://www.biodiversity-science.net/fileup/PDF/2020268-5.pdf

附录6 在未来气候变化情景下, 3 种黄耆属植物与其传粉熊蜂的地理范围相对于历史地理范围的变化及损失范围变化百分比 Appendix 6 Relative range contraction or expansion (percentage) for three Astragalus species and pollinating bumblebees under future climate change scenarios with respect to their near current estimate ranges and lost range https://www.biodiversity-science.net/fileup/PDF/2020268-6.pdf 
施雨含, 任宗昕, 王维嘉, 徐金金, 刘杰, 赵延会, 王红 (2021) 中国-喜马拉雅三种黄耆属植物与其传粉熊蜂 的空间分布预测. 生物多样性, 29, 759-769. https://www.biodiversity-science.net/CN/10.17520/biods.2020268

\section{附录 13 种黄耆属植物传粉昆虫的种类及数量}

Appendix 1 Species and number of pollinators of three Astragalus species

\begin{tabular}{|c|c|c|}
\hline $\begin{array}{l}\text { 植物 } \\
\text { Plant }\end{array}$ & $\begin{array}{l}\text { 传粉昆虫种类 } \\
\text { Pollinator }\end{array}$ & $\begin{array}{l}\text { 数量 } \\
\text { Number }\end{array}$ \\
\hline \multirow[t]{10}{*}{ 弯齿黄耆 Astragalus camptodontus } & 橘尾熊蜂 Bombus friseanus & 179 \\
\hline & 中华蜜蜂 Apis cerana & 31 \\
\hline & 小雅熊蜂 Bombus lepidus & 12 \\
\hline & 熊蜂属一种 Bombus sp. & 10 \\
\hline & 兴熊蜂 Bombus impetuosus & 4 \\
\hline & 灰熊蜂 Bombus grahami & 2 \\
\hline & 疏熊蜂 Bombus remotus & 2 \\
\hline & 南熊蜂 Bombus securus & 1 \\
\hline & 眠熊蜂 Bombus hypnorum & 1 \\
\hline & 贞洁熊蜂 Bombus infrequens & 1 \\
\hline \multirow[t]{6}{*}{ 黑毛黄耆 Astragalus pullus } & 橘尾熊蜂 Bombus friseanus & 277 \\
\hline & 中华蜜蜂 Apis cerana & 193 \\
\hline & 小雅熊蜂 Bombus lepidus & 55 \\
\hline & 兴熊蜂 Bombus impetuosus & 2 \\
\hline & 白背熊蜂 Bombus festivus & 1 \\
\hline & 疏熊蜂 Bombus remotus & 1 \\
\hline \multirow[t]{19}{*}{ 笔直黄耆 Astragalus strictus } & 红束熊蜂 Bombus rufofasciatus & 419 \\
\hline & 橘尾熊蜂 Bombus friseanus & 274 \\
\hline & 拉达克熊蜂 Bombus ladakhensis & 152 \\
\hline & 凸污熊蜂 Bombus convexus & 89 \\
\hline & 小雅熊蜂 Bombus lepidus & 87 \\
\hline & 稳纹熊蜂 Bombus waltoni & 75 \\
\hline & 兴熊蜂 Bombus impetuosus & 30 \\
\hline & 克什米尔熊蜂 Bombus kashmirensis & 21 \\
\hline & 饰带熊蜂 Bombus lemniscatus & 21 \\
\hline & 眠熊蜂 Bombus hypnorum & 18 \\
\hline & 伪猛熊蜂 Bombus personatus & 17 \\
\hline & 长翅熊蜂 Bombus longipennis & 7 \\
\hline & 斯科熊蜂 Bombus skorikovi & 3 \\
\hline & 疏熊蜂 Bombus remotus & 2 \\
\hline & 昆仑熊蜂 Bombus keriensis & 2 \\
\hline & 西藏熊蜂 Bombus tibetanus & 1 \\
\hline & 白背熊蜂 Bombus festivus & 1 \\
\hline & 圣熊蜂 Bombus religiosus & 1 \\
\hline & 布氏熊蜂 Bombus branickii & 1 \\
\hline
\end{tabular}

Bombus sp. 为待发表新种 
施雨含, 任宗昕, 王维嘉, 徐金, 刘杰, 赵延会, 王红 (2021) 中国-喜马拉雅三种黄耆属植物与其传粉熊蜂 的空间分布预测. 生物多样性, 29, 759-769. https://www.biodiversity-science.net/CN/10.17520/biods.2020268

附录 2 高山草甸样地信息

Appendix 2 Information of alpine meadow plots

\begin{tabular}{|c|c|c|c|}
\hline $\begin{array}{l}\text { 样地 } \\
\text { Plots }\end{array}$ & $\begin{array}{l}\text { 名称 } \\
\text { Name }\end{array}$ & $\begin{array}{l}\text { 经纬度 } \\
\text { Longitude, Latitude }\end{array}$ & $\begin{array}{l}\text { 海拔 } \\
\text { Elevation (m) }\end{array}$ \\
\hline \multicolumn{4}{|c|}{ 丽江 Lijiang } \\
\hline 1 & 文海(WH) & $100^{\circ} 10^{\prime} \mathrm{E}, 26^{\circ} 58^{\prime} \mathrm{N}$ & 3,000 \\
\hline 2 & 水库(SK) & $100^{\circ} 07^{\prime} \mathrm{E}, 26^{\circ} 32^{\prime} \mathrm{N}$ & 3,260 \\
\hline 3 & 金矿(JK) & $100^{\circ} 13^{\prime} \mathrm{E}, 27^{\circ} 01^{\prime} \mathrm{N}$ & 3,280 \\
\hline 4 & 丽江高山植物园工作站(GZZ) & $100^{\circ} 06^{\prime} \mathrm{E}, 26^{\circ} 35^{\prime} \mathrm{N}$ & 3,300 \\
\hline \multicolumn{4}{|c|}{ 香格里拉 Xiang-Gelila } \\
\hline & 白马雪山L (BMXSL) & $99^{\circ} 09^{\prime} \mathrm{E}, 28^{\circ} 17^{\prime} \mathrm{N}$ & 3,331 \\
\hline \multicolumn{4}{|c|}{ 西藏 Tibet } \\
\hline 1 & 东达山M (DDSM) & $98^{\circ} 10^{\prime} \mathrm{E}, 29^{\circ} 35^{\prime} \mathrm{N}$ & 3,886 \\
\hline 2 & 珠角拉山M (ZJLSM) & $96^{\circ} 38^{\prime} \mathrm{E}, 29^{\circ} 29^{\prime} \mathrm{N}$ & 3,899 \\
\hline 3 & 米拉山M (MLSM) & $96^{\circ} 52^{\prime} \mathrm{E}, 31^{\circ} 06^{\prime} \mathrm{N}$ & 3,907 \\
\hline 4 & 然乌 $\mathrm{M}(\mathrm{RWM})$ & $96^{\circ} 38^{\prime} \mathrm{E}, 29^{\circ} 29^{\prime} \mathrm{N}$ & 3,910 \\
\hline 5 & 白马雪山M (BMXSM) & $99^{\circ} 03^{\prime} \mathrm{E}, 28^{\circ} 20^{\prime} \mathrm{N}$ & 3,953 \\
\hline 6 & 矮拉山M (ALSM) & $98^{\circ} 28^{\prime} \mathrm{E}, 31^{\circ} 36^{\prime} \mathrm{N}$ & 3,986 \\
\hline 7 & 雀儿山M (QESM) & $98^{\circ} 51^{\prime} \mathrm{E}, 31^{\circ} 57^{\prime} \mathrm{N}$ & 4,008 \\
\hline 8 & 安久拉山H (AJLSH) & $96^{\circ} 43^{\prime} \mathrm{E}, 29^{\circ} 39^{\prime} \mathrm{N}$ & 4,527 \\
\hline 9 & 业拉山H (YLSH) & $97^{\circ} 17^{\prime} \mathrm{E}, 30^{\circ} 09^{\prime} \mathrm{N}$ & 4,547 \\
\hline 10 & 东达山H (DDSH) & $98^{\circ} 06^{\prime} \mathrm{E}, 29^{\circ} 42^{\prime} \mathrm{N}$ & 4,561 \\
\hline 11 & 米拉山H (MLSH) & $92^{\circ} 20^{\prime} \mathrm{E}, 29^{\circ} 51^{\prime} \mathrm{N}$ & 4,760 \\
\hline
\end{tabular}

西藏居群中 $\mathrm{L}(\mathrm{Low})$ 为低海拔段居群, $\mathrm{M}$ (Middle)为中海拔段居群, $\mathrm{H}(\mathrm{High})$ 为高海拔段居群。 
施雨含, 任宗昕, 王维嘉, 徐金金, 刘杰, 赵延会, 王红 (2021) 中国-喜马拉雅三种黄耆属植物与其传粉熊蜂 的空间分布预测. 生物多样性, 29, 759-769. https://www.biodiversity-science.net/CN/10.17520/biods.2020268

\section{附录 3 WorldClim 的环境变量}

Appendix 3 Environment variable from WorldClim

\begin{tabular}{|c|c|}
\hline $\begin{array}{l}\text { 数据类型 } \\
\text { Data type }\end{array}$ & $\begin{array}{l}\text { 生态因子 } \\
\text { Ecological factor }\end{array}$ \\
\hline \multirow[t]{19}{*}{ 气候变量 Climate variables } & 年平均气温 Annual mean temperature (Bio 1) \\
\hline & 昼夜温差月均值 Mean diurnal range (Mean of monthly (max temp-min temp)) (Bio 2) \\
\hline & 昼夜温差与年温差比值 Isothermality $(\mathrm{BIO} 2 / \mathrm{BIO} 7)(\times 100))(\mathrm{Bio} 3)$ \\
\hline & 温度变化方差 Temperature seasonality (standard deviation × 100) (Bio 4) \\
\hline & 最热月份最高温 Max temperature of warmest month (Bio 5) \\
\hline & 最冷月份最高温 Min temperature of coldest month (Bio 6) \\
\hline & 年温度变化范围 Temperature annual range (BIO5-BIO6) (Bio 7) \\
\hline & 最湿季均温 Mean temperature of wettest quarter (Bio 8) \\
\hline & 最干季均温 Mean temperature of driest quarter (Bio 9) \\
\hline & 最热季均温 Mean temperature of warmest quarter (Bio 10) \\
\hline & 最冷季均温 Mean temperature of coldest quarter (Bio 11) \\
\hline & 年均降水量 Annual precipitation (Bio 12) \\
\hline & 最湿月降水量 Precipitation of wettest month (Bio 13) \\
\hline & 最干月降水量 Precipitation of driest month (Bio 14) \\
\hline & 湿度变化方差 Precipitation seasonality (coefficient of variation) (Bio 15) \\
\hline & 最湿季降水量 Precipitation of wettest quarter (Bio 16) \\
\hline & 最干季降水量 Precipitation of driest quarter (Bio 17) \\
\hline & 最热季平均降水量 Precipitation of warmest quarter (Bio 18) \\
\hline & 最冷季平均降水量 Precipitation of coldest quarter (Bio 19) \\
\hline \multirow[t]{3}{*}{ 地形变量 Topographic variables } & 海拔 Elevation \\
\hline & 坡度 Slope \\
\hline & 坡向 Aspect \\
\hline
\end{tabular}


施雨含, 任宗昕, 王维嘉, 徐金金, 刘杰, 赵延会, 王红 (2021) 中国-喜马拉雅三种黄耆属植物与其传粉熊蜂的空间分布预测. 生物多样性, 29, 759-769. https://www.biodiversityscience.net/CN/10.17520/biods.2020268

附录 419 个气候变量的相关性矩阵

Appendix 4 Correlation matrix of 19 climate variables

\begin{tabular}{|c|c|c|c|c|c|c|c|c|c|c|c|c|c|c|c|c|c|c|c|}
\hline & Bio1 & Bio2 & Bio3 & Bio4 & Bio5 & Bio6 & Bio7 & Bio8 & Bio9 & Bio10 & Bio11 & Bio12 & Bio13 & Bio14 & Bio15 & Bio16 & Bio17 & Bio18 & Bio19 \\
\hline Bio1 & 1.00 & 0.56 & 0.70 & 0.38 & 0.77 & 0.13 & 0.46 & 0.77 & 0.65 & 0.82 & 0.47 & 0.71 & 0.72 & 0.46 & 0.61 & 0.72 & 0.51 & 0.58 & 0.55 \\
\hline Bio2 & 0.56 & 1.00 & 0.96 & 0.95 & 0.95 & -0.73 & 0.98 & 0.87 & -0.10 & 0.91 & -0.44 & 0.63 & 0.63 & 0.48 & 0.95 & 0.63 & 0.51 & 0.62 & 0.56 \\
\hline Bio3 & 0.70 & 0.96 & 1.00 & 0.84 & 0.95 & -0.55 & 0.90 & 0.90 & 0.07 & 0.93 & -0.22 & 0.76 & 0.76 & 0.54 & 0.95 & 0.76 & 0.58 & 0.72 & 0.62 \\
\hline Bio4 & 0.38 & 0.95 & 0.84 & 1.00 & 0.88 & -0.86 & 0.99 & 0.81 & -0.30 & 0.84 & -0.63 & 0.54 & 0.52 & 0.51 & 0.86 & 0.52 & 0.52 & 0.56 & 0.54 \\
\hline Bio5 & 0.77 & 0.95 & 0.95 & 0.88 & 1.00 & -0.53 & 0.92 & 0.94 & 0.13 & 0.99 & -0.20 & 0.72 & 0.72 & 0.57 & 0.91 & 0.72 & 0.61 & 0.66 & 0.65 \\
\hline Bio6 & 0.13 & -0.73 & -0.55 & -0.86 & -0.53 & 1.00 & -0.82 & -0.46 & 0.69 & -0.45 & 0.93 & -0.20 & -0.19 & -0.26 & -0.63 & -0.18 & -0.25 & -0.30 & -0.25 \\
\hline Bio7 & 0.46 & 0.98 & 0.90 & 0.99 & 0.92 & -0.82 & 1.00 & 0.85 & -0.23 & 0.88 & -0.57 & 0.58 & 0.58 & 0.51 & 0.91 & 0.57 & 0.53 & 0.59 & 0.55 \\
\hline Bio8 & 0.77 & 0.87 & 0.90 & 0.81 & 0.94 & -0.46 & 0.85 & 1.00 & 0.05 & 0.95 & -0.13 & 0.77 & 0.77 & 0.58 & 0.89 & 0.76 & 0.62 & 0.73 & 0.55 \\
\hline Bio9 & 0.65 & -0.10 & 0.07 & -0.30 & 0.13 & 0.69 & -0.23 & 0.05 & 1.00 & 0.20 & 0.83 & 0.19 & 0.20 & 0.03 & -0.07 & 0.20 & 0.07 & 0.01 & 0.24 \\
\hline Bio10 & 0.82 & 0.91 & 0.93 & 0.84 & 0.99 & -0.45 & 0.88 & 0.95 & 0.20 & 1.00 & -0.12 & 0.75 & 0.74 & 0.58 & 0.89 & 0.74 & 0.62 & 0.68 & 0.66 \\
\hline Bio11 & 0.47 & -0.44 & -0.22 & -0.63 & -0.20 & 0.93 & -0.57 & -0.13 & 0.83 & -0.12 & 1.00 & 0.08 & 0.10 & -0.10 & -0.32 & 0.11 & -0.06 & -0.06 & -0.04 \\
\hline Bio12 & 0.71 & 0.63 & 0.76 & 0.54 & 0.72 & -0.20 & 0.58 & 0.77 & 0.19 & 0.75 & 0.08 & 1.00 & 0.97 & 0.72 & 0.69 & 0.98 & 0.77 & 0.94 & 0.70 \\
\hline
\end{tabular}


施雨含, 任宗昕, 王维嘉, 徐金金, 刘杰, 赵延会, 王红 (2021) 中国-喜马拉雅三种黄耆属植物与其传粉熊蜂的空间分布预测. 生物多样性, 29, 759-769. https://www.biodiversityscience.net/CN/10.17520/biods.2020268

\begin{tabular}{|c|c|c|c|c|c|c|c|c|c|c|c|c|c|c|c|c|c|c|c|}
\hline Bio13 & 0.72 & 0.63 & 0.76 & 0.52 & 0.72 & -0.19 & 0.58 & 0.77 & 0.20 & 0.74 & 0.10 & 0.97 & 1.00 & 0.59 & 0.73 & 1.00 & 0.65 & 0.90 & 0.59 \\
\hline Bio14 & 0.46 & 0.48 & 0.54 & 0.51 & 0.57 & -0.26 & 0.51 & 0.58 & 0.03 & 0.58 & -0.10 & 0.72 & 0.59 & 1.00 & 0.43 & 0.60 & 0.99 & 0.66 & 0.85 \\
\hline Bio15 & 0.61 & 0.95 & 0.95 & 0.86 & 0.91 & -0.63 & 0.91 & 0.89 & -0.07 & 0.89 & -0.32 & 0.69 & 0.73 & 0.43 & 1.00 & 0.72 & 0.46 & 0.68 & 0.50 \\
\hline Bio16 & 0.72 & 0.63 & 0.76 & 0.52 & 0.72 & -0.18 & 0.57 & 0.76 & 0.20 & 0.74 & 0.11 & 0.98 & 1.00 & 0.60 & 0.72 & 1.00 & 0.66 & 0.91 & 0.60 \\
\hline Bio17 & 0.51 & 0.51 & 0.58 & 0.52 & 0.61 & -0.25 & 0.53 & 0.62 & 0.07 & 0.62 & -0.06 & 0.77 & 0.65 & 0.99 & 0.46 & 0.66 & 1.00 & 0.70 & 0.88 \\
\hline Bio18 & 0.58 & 0.62 & 0.72 & 0.56 & 0.66 & -0.30 & 0.59 & 0.73 & 0.01 & 0.68 & -0.06 & 0.94 & 0.90 & 0.66 & 0.68 & 0.91 & 0.70 & 1.00 & 0.59 \\
\hline Bio19 & 0.55 & 0.56 & 0.62 & 0.54 & 0.65 & -0.25 & 0.55 & 0.55 & 0.24 & 0.66 & -0.04 & 0.70 & 0.59 & 0.85 & 0.50 & 0.60 & 0.88 & 0.59 & 1.00 \\
\hline
\end{tabular}


施雨含, 任宗昕, 王维嘉, 徐金金, 刘杰, 赵延会, 王红 (2021) 中国-喜马拉雅三种黄耆属植物与其传粉熊蜂的空间分布预测. 生物多样性, 29, 759-769. https://www.biodiversity-science.net/CN/10.17520/biods.2020268

附录 5 用于 MaxEnt 模型的相关气候变量

Appendix 5 Climate variables for MaxEnt model

\begin{tabular}{ll}
\hline $\begin{array}{l}\text { 物种 } \\
\text { Species }\end{array}$ & $\begin{array}{l}\text { 气候变量 } \\
\text { Climate variables }\end{array}$ \\
\hline 弯齿黄耆 Astragalus camptodontus & Bio1、Bio3、Bio11、Bio12、Bio19 \\
黑毛黄耆 Astragalus pullus & Bio3、Bio11、Bio17、Bio18 \\
笔直黄耆 Astragalus strictus & Bio1、Bio3、Bio9、Bio17 \\
橘尾熊蜂 Bombus friseanus & Bio4、Bio11、Bio18、Bio19 \\
红束熊蜂 Bombus rufofasciatus & Bio1、Bio2、Bio9、Bio12、Bio17 \\
\hline
\end{tabular}


施雨含, 任宗昕, 王维嘉, 徐金金, 刘杰, 赵延会, 王红 (2021) 中国-喜马拉雅三种黄耆属植物与其传粉熊蜂的空间分布预测. 生物多样性, 29, 759-769. https://www.biodiversity-science.net/CN/10.17520/biods.2020268

附录 6 在未来气候变化情景下, 3 种黄耆属植物与其传粉熊蜂的地理范围相对于历史地理范围的变化及损 失范围变化百分比

Appendix 6 Relative range contraction or expansion (percentage) for three Astragalus species and pollinating bumblebees under future climate change scenarios with respect to their near current estimate ranges and lost range

\begin{tabular}{|c|c|c|c|c|c|c|}
\hline \multirow[t]{3}{*}{$\begin{array}{l}\text { 物种 } \\
\text { Species }\end{array}$} & \multirow{2}{*}{\multicolumn{2}{|c|}{$\begin{array}{c}\text { 收缩范围 } \\
\text { Area to be lost (\%) }\end{array}$}} & \multicolumn{4}{|c|}{$\begin{array}{c}\text { 总地理范围变化 } \\
\text { Change in total range size }(\%) \\
\end{array}$} \\
\hline & & & \multicolumn{2}{|c|}{ 完全扩散 Full dispersal } & \multicolumn{2}{|c|}{ 不扩散 No dispersal } \\
\hline & $\operatorname{ssp} 245$ & ssp585 & ssp245 & ssp585 & $\operatorname{ssp} 245$ & ssp585 \\
\hline 弯齿黄耆 Astragalus & 16.20 & 30.91 & 63.29 & 120.25 & -15.89 & -31.59 \\
\hline \multicolumn{7}{|l|}{ camptodontus } \\
\hline 黑毛黄者 Astragalus pullus & 15.43 & 15.03 & 14.49 & 87.57 & -14.87 & -15.02 \\
\hline 笔直黄者 Astragalus strictus & 4.15 & 2.04 & 32.36 & 45.53 & -4.06 & -1.86 \\
\hline 橘尾熊蜂 Bombus friseanus & 2.13 & 11.53 & 27.34 & 34.72 & -1.50 & -11.41 \\
\hline 红束熊蜂 Bombus & 10.05 & 12.32 & 53.90 & 96.89 & -10.20 & -12.54 \\
\hline rufofasciatus & & & & & & \\
\hline
\end{tabular}

